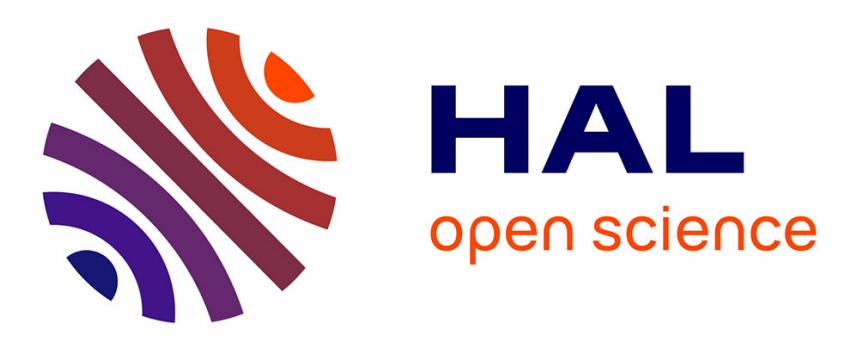

\title{
A numerical method for shape and topology optimization for semilinear elliptic equation
}

Jean-François Scheid, Jan Sokolowski, Katarzyna Szulc

\section{To cite this version:}

Jean-François Scheid, Jan Sokolowski, Katarzyna Szulc. A numerical method for shape and topology optimization for semilinear elliptic equation. MMAR 2010: 15th International Conference on Methods \& Models in Automation \& Robotics, Aug 2010, Miedzyzdroje, Poland. pp.290-295, 10.1109/MMAR.2010.5587220 . hal-00589922

\section{HAL Id: hal-00589922 \\ https://hal.science/hal-00589922}

Submitted on 22 Jan 2021

HAL is a multi-disciplinary open access archive for the deposit and dissemination of scientific research documents, whether they are published or not. The documents may come from teaching and research institutions in France or abroad, or from public or private research centers.
L'archive ouverte pluridisciplinaire HAL, est destinée au dépôt et à la diffusion de documents scientifiques de niveau recherche, publiés ou non, émanant des établissements d'enseignement et de recherche français ou étrangers, des laboratoires publics ou privés. 


\title{
A numerical method for shape and topology optimization for semilinear elliptic equation
}

\author{
Jean-François Scheid, Jan Sokołowski and Katarzyna Szulc \\ Université Henri Poincaré Nancy I \\ Institut Elie Cartan, Laboratoire de Mathématiques \\ BP. 239, 54506 Vandoeuvre-lès-Nancy, France \\ Email: \{Jean-Francois.Scheid, Jan.Sokolowski, Katarzyna.Szulc\}@iecn.u-nancy.fr
}

\begin{abstract}
Shape optimization problem for semilinear elliptic equation is considered. There is an optimal solution which is computed by the Levelset method. To this end the shape derivative of the functional is evaluated. In order to predict the topology changes the topological derivative is employed. Numerical results confirm that the proposed framework for numerical solution of shape optimization problems is efficient.
\end{abstract}

\section{INTRODUCTION}

In this paper we are going to present a numerical method for shape optimisation problem of an integral functional associated to a non-linear boundary value problem. This method uses the shape derivative and the topological derivative of the shape functional. Geometrical evolution of the domain is controlled by the level set method. Topological changes are possible due to an application of the topological derivative. The form of topological derivative is presented in [8], [34].

We introduce the model problem. Let $B$ and $D$ be two bounded open subsets of $\mathbb{R}^{2}$ such that $B \Subset D$ For any open set $\omega \subset \mathbb{R}^{2}$, we denote $\# \omega$ the number of connected components of $\bar{\omega}$ and we consider the set of admissible domains

$$
\begin{array}{r}
\mathcal{U}_{a d}=\{\Omega=D \backslash \bar{\omega}: \omega \text { open set, } \omega \subset B, \# \omega \leq k, \\
\left.\mathcal{H}^{1}(\partial \omega) \leq C\right\}
\end{array}
$$

with $\mathcal{H}^{1}(\partial \omega)$ the length of the boundaries and $C$ given integer. We denote $\partial D=\Gamma$ the boundary of $D$ and $\partial \Omega=\Gamma \cup \partial \omega$. We consider a shape optimisation problem for the following semilinear boundary value problem

$$
\left\{\begin{aligned}
-\Delta u+u^{3} & =f \quad \text { in } \quad \Omega, \\
u & =0 \text { on } \Gamma, \\
\frac{\partial u}{\partial n} & =0 \text { on } \partial \omega,
\end{aligned}\right.
$$

where $f \subset C^{\infty}(D)$ and $n$ is the unit outward normal vector to $\partial \Omega$, (cf. Fig.2).

Given a function $z_{d}$ defined in $D$, the shape functional is given as follows

$$
J(\Omega):=\frac{1}{2} \int_{\Omega}\left(u-z_{d}\right)^{2} d x .
$$

We are interested in the following shape optimisation problem

$$
\min \left\{J(\Omega): \Omega \in \mathcal{U}_{a d}\right\}
$$

associated with (2).

We denote that $H_{\Gamma}^{1}(\Omega)=\left\{v \in H^{1}(\Omega): v=0\right.$ on $\left.\Gamma\right\}$.

Lemma 1.1: For any given $f \in L^{2}(D)$ there exists a unique solution $u \in H_{\Gamma}^{1}(\Omega) \cap L^{4}(\Omega)$ to boundary value problem (2). The solution $u$ minimizes the energy functional

$$
I(\varphi)=\frac{1}{2} \int_{\Omega}|\nabla \varphi|^{2}+\frac{1}{4} \int_{\Omega}|\varphi|^{4}-\int_{\Omega} f \varphi
$$

over the space $H_{\Gamma}^{1}(\Omega) \cap L^{4}(\Omega)$.

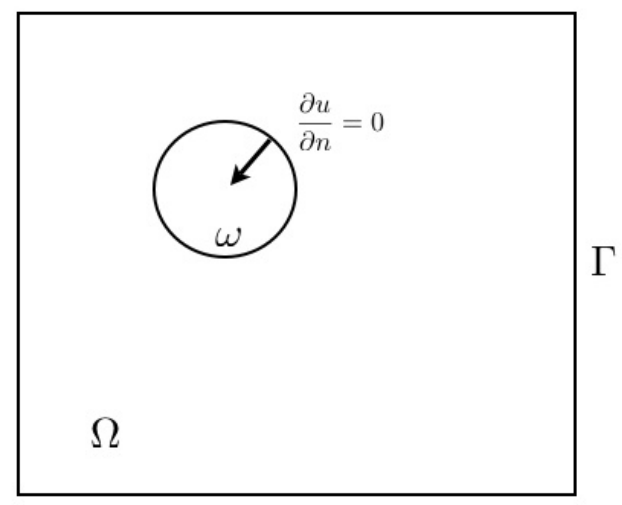

Fig. 1. Domain $\Omega=D \backslash \bar{\omega}$.

We are going to solve numerically the shape optimization problem for (2). In our notation $D$ is a hold-all domain, and $\omega$ stands for a hole, which is opened in $D$, the location of the hole is determined taking into account the topological derivative of a specific shape functional to be minimized over the family of admissible domaines.

We proceed in the following way. First, in section II the existence of an optimal domain within the class (family) of admissible domains is established. Then, the shape derivation of the functional (3) is performed in sections III. In the subsequent section the topological derivative of the shape functional is given [8]. Finally, numerical results for the shape optimization problem are presented.

\section{EXISTENCE OF OPTIMAL DOMAINS}

We are going to minimize the functional (3) with respect to $\Omega$. We refer to [2], [3] for the existence result of an optimal 
domain in (4) for the linear elliptic problem. In this paper we can adopt the results of [2], [3] which is possible because the boundary $\Gamma$ is fixed.

Given a set $D \subset \mathbb{R}^{2}$ and $f \in L^{2}(D)$, for every open set $\Omega \subset D$ we denote by $u_{\Omega}$ the weak variational solution of

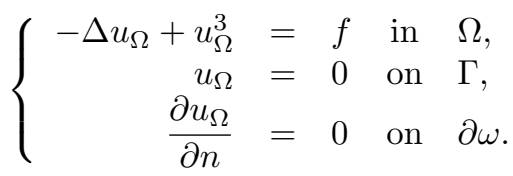

Denoting by $\tilde{u}_{\Omega}$ the extension by zero of $u_{\Omega}$ to an element of $L^{4}(D)$, we study the continuity of the mapping $\Omega \mapsto$ $\tilde{u}_{\Omega} \in L^{2}(D)$, if the family of domains is endowed with the Hausdorff complementary topology. The starting point is the following two dimensional result:

If $\Omega_{n}$ converges in the Hausdorff complementary topology (simply denoted $H^{c}$ ) to some $\Omega$ such that the number of connected components of $D \backslash \Omega_{n}$ and the length of the boundaries $\mathcal{H}^{1}\left(\omega_{n}\right)$ are uniformly bounded, then $\tilde{u}_{\Omega_{n}}$ in $H^{1}$ converge to $\tilde{u}_{\Omega}$. More precisely, we prove that if $\Omega_{n}$ converges in the Hausdorff complementary topology to $\Omega$, and the number of connected components of the complements $D \backslash \Omega_{n}$ is uniformly bounded, then for every $f \in L^{2}(D)$ there is the convergence of solutions $\tilde{u}_{\Omega_{n}} \rightarrow \tilde{u}_{\Omega}$ if and only if there is the convergence of the Lebesque measures $\left|\Omega_{n}\right| \rightarrow|\Omega|$. We apply this result in order to prove the existence of an optimal domain which minimizes the shape functional

$$
J(\Omega):=\frac{1}{2} \int_{\Omega}\left(u_{\Omega}-z_{d}\right)^{2} d x
$$

depending on $u_{\Omega}$.

The weak solution of problem (6) is the unique function $u_{\Omega} \in H^{1}(\Omega) \cap L^{4}(\Omega), u=0$ on $\Gamma$, satisfying

$$
\int_{\Omega}\left(\nabla u_{\Omega} \nabla v+u_{\Omega}^{3} v\right) d x=\int_{\Omega} f v d x \quad \forall v \in H^{1}(\Omega), v=0 \text { on } \Gamma .
$$

In order to compare the solutions of problem (6) defined on two different domains, all functions of $H^{1}(\Omega)$ are extended by zero to elements of $L^{2}(D)$ as well as their gradients. Hence, for every $u \in H^{1}(\Omega)$ we denote by $\tilde{u}$ an element of $L^{2}(D)$, defined as $\tilde{u}(x)=u(x)$ if $x \in \Omega$ and $\tilde{u}(x)=0$ if $x \in D \backslash \Omega$. The gradient of $u$ is extended in the same way to an element of $L^{2}\left(D, \mathbb{R}^{2}\right)$. We write $\tilde{\nabla} u(x)=\nabla u(x)$ if $x \in \Omega$ and $\tilde{\nabla} u(x)=$ 0 if $x \in D \backslash \Omega$. In this way, $H^{1}(\Omega)$ can be identified with a closed subspace in $L^{2}(D) \times L^{2}\left(D, \mathbb{R}^{2}\right)$.

Given a sequence of elements $\left\{\Omega_{n}\right\}_{n \in \mathbb{N}}$ in $\mathcal{U}_{a d}$, it is said that $H^{1}\left(\Omega_{n}\right)$ converge in the sense of Mosco to $H^{1}(\Omega)$ if

M1) For each $\phi \in H^{1}(\Omega)$ there exists a sequence $\phi_{n} \in$ $H^{1}\left(\Omega_{n}\right)$ such that $\tilde{\phi}_{n}$ converges strongly in $L^{2}(D)$ to $\tilde{\phi}$ and $\tilde{\nabla} \phi_{n}$ converges strongly in $L^{2}\left(D, \mathbb{R}^{2}\right)$ to $\tilde{\nabla} \phi$;

M2) For every sequence $\phi_{k} \in H^{1}\left(\Omega_{n_{k}}\right)$ such that $\left(\tilde{\phi}_{k}, \tilde{\nabla} \phi_{k}\right)$ is weakly convergent in $L^{2}(D) \times L^{2}\left(D, \mathbb{R}^{2}\right)$ to $\left(u, v_{1}, v_{2}\right)$ we have that $\left(u, v_{1}, v_{2}\right)=0$ a.e. in $D \backslash \Omega$ and $\nabla u=\left(v_{1}, v_{2}\right)$ in $\Omega$, this equality is understood in the sense of distributions.
In order to simplify notations, for $u_{n} \in H^{1}\left(\Omega_{n}\right)$ and $u \in H^{1}(\Omega)$, we write $u_{n} \leadsto u$ instead of $\left(\tilde{u}_{n}, \tilde{\nabla} u_{n}\right) \stackrel{L^{2}(D) \times L^{2}\left(D, \mathbb{R}^{2}\right)}{\longrightarrow}(\tilde{u}, \tilde{\nabla} u)$.

We consider a family of domains compact with respect to the Mosco convergence. Shape functional $J(\Omega)$ is minimized with respect to $\Omega$, where $u_{\Omega}$ is given by (6). The family of admissible domains denoted by $\mathcal{U}_{a d}$ is given by (1). In order to show the existence of a minimizer $\Omega^{*} \in \mathcal{U}_{a d}$ to the problem

$$
J\left(\Omega^{*}\right)=\inf _{\Omega \in \mathcal{U}_{a d}} J(\Omega)
$$

we are going to use the standard approach. Assume that $\left\{\Omega_{n}\right\}$ is a minimizing sequence for the shape optimization problem.

The existence result for the shape optimization problem is established under the following assumptions on the minimizing sequence $\left\{\Omega_{n}\right\}$.

H1. $\Omega_{n}=D \backslash \bigcup_{i=1}^{N} \omega_{n}^{i}$ and $\mathcal{H}_{c}\left(\Omega_{n}\right):=\mathcal{H}\left(\bigcup_{i=1}^{N} \omega_{n}^{i}\right) \rightarrow$
$\mathcal{H}_{c}\left(\Omega_{\infty}\right):=\mathcal{H}\left(\bigcup_{i=1}^{N} \omega_{\infty}^{i}\right)$

$\mathrm{H} 2$. there is the Mosco convergence of the spaces $H^{1}\left(\Omega_{n}\right)$ to $H^{1}(\Omega)$ denoted by $H^{1}\left(\Omega_{n}\right) \stackrel{M}{\longrightarrow} H^{1}(\Omega)$ (cf. [3])

Remark 2.1: Assumption H2. implies the Mosco convergence of $H^{1}\left(\Omega_{n}\right) \cap L^{p}\left(\Omega_{n}\right)$ to $H^{1}\left(\Omega_{\infty}\right) \cap L^{p}\left(\Omega_{\infty}\right)$ for all $p<\infty$. This property can be established by using a dense subset in (M1) of functions of the form $u_{M}(x)=$ $\inf \left(u_{+}(x), M\right)-\inf \left(u_{-}(x), M\right)$ in $H^{1}\left(\Omega_{\infty}\right), M<\infty$, where $u_{+}(x)=\sup \{u(x), 0\} ; u_{-}(x)=\sup \{-u(x), 0\}$.

Theorem 2.2: There exists a solution to the problem (9) in the class of admissible domains (1).

In order to proof this theorem it is sufficient to proof the following:

Theorem 2.3: Let $l \in \mathbb{N}$ be fixed, and $\Omega_{n}, \Omega \in \mathcal{U}_{a d}$ such that $\Omega_{n} \rightarrow \Omega$. Then for every $f \in L^{2}(D)$ we have $u_{\Omega_{n}} \leadsto u_{\Omega}$ if and only if $\left|\Omega_{n}\right| \rightarrow|\Omega|$.

The proof of this theorem in the case of linear boundary value problems, can be found in [3]. In order to adopt this result for the semi-linear problem (6) we can establish the convergence in the sense of Mosco of the associated Sobolev spaces $H^{1}(\Omega) \cap L^{p}(\Omega)$, for all $p<\infty$. The relation between the continuity with respect to the geometric domain for problem (6) and the convergence in the sense of Mosco of the Sobolev spaces is given in the following proposition.

Proposition 2.1: Let $\Omega_{n}, \Omega \in \mathcal{U}_{a d}$ be such that $H^{1}\left(\Omega_{n}\right)$ converge in the sense of Mosco to $H^{1}(\Omega)$. Then for every $f \in L^{2}(D)$ we have that $u_{\Omega_{n}} \leadsto u_{\Omega}$.

The proof of the Proposition 2.1 can be found in [34]

\section{Shape AND TOPOLOGICAL DERIVATIVES}

We recall briefly the formal way to derive the equation and the boundary conditions for the shape derivative $u^{\prime}$ [32]. The shape derivative of $J(\Omega)$ is determined by the usual formula

$$
d J(\Omega ; V)=\lim _{t \rightarrow 0} \frac{1}{t}\left(J\left(\Omega_{t}\right)-J(\Omega)\right) .
$$


The set $\Omega_{t}$, being a small perturbation of $\Omega$, is defined as an image of $\Omega$ by the application $T_{t}: \mathbb{R}^{2} \rightarrow \mathbb{R}^{2}$ given by the flux of a smooth vector field $V: \mathbb{R} \times \mathbb{R}^{2} \rightarrow \mathbb{R}^{2}$, the solution to

$$
\left\{\begin{aligned}
\frac{d x}{d t}(t) & =V(t, x(t)) \\
x(0) & =X
\end{aligned}\right.
$$

It means that $T_{t}(X)=x(t, X)$ for $t>0$ and $X \in \Omega$. The limit (10) defines the derivative of the functional $J(\Omega)$ in the direction of vector field $V$. From (3) we get

$$
d J(\Omega ; V)=\int_{\Omega}\left(u-z_{d}\right) u^{\prime}+\int_{\partial \omega}\left(u-z_{d}\right)^{2}\langle V, n\rangle,
$$

where the shape derivative $u^{\prime}$ of the solution to (2) is determined below and $V=0$ on $\Gamma$.

It is straightforward to obtain for $u^{\prime}$ the boundary conditions on $\Gamma$ and the equation in $\Omega$,

$$
\begin{aligned}
u^{\prime}=0 & \text { on } \quad \Gamma, \\
-\Delta u^{\prime}+3 u^{2} u^{\prime}=0 & \text { in } \Omega .
\end{aligned}
$$

The boundary condition on $\partial \omega$ is established in (3) to complete the system (13). To this end, we recall that $u=0$ on $\Gamma$, then the material derivative reads $\dot{u}=u^{\prime}+\nabla u \cdot V=0$. Thus, the shape derivative $u^{\prime}=-\nabla u \cdot V=-\frac{\partial u}{\partial n}\langle V, n\rangle$. By construction, the vector field $V$ vanishes on $\Gamma$.

We use the decomposition of the gradient on the boundary into the tangential and normal components $\nabla u=\nabla_{\Gamma} u+\frac{\partial u}{\partial n} n$. The material derivative is defined in the standard way

$$
\frac{d}{d t} u_{t}(x(t))=\dot{u}=u^{\prime}+\nabla u \cdot V .
$$

We refer the reader to [32] for further details.

\section{A. Variational formulation of the semilinear boundary value problem}

The mixed boundary value problem is written in the form of the integral identity:

Find $u \in H_{\Gamma}^{1}\left(\Omega_{t}\right) \cap L^{4}\left(\Omega_{t}\right)$ such that

$$
\int_{\Omega_{t}} \nabla u \nabla \varphi+\int_{\Omega_{t}} u^{3} \varphi=\int_{\Omega_{t}} f \varphi \quad \forall \varphi \in H_{\Gamma}^{1}\left(\Omega_{t}\right) \cap L^{4}\left(\Omega_{t}\right)
$$

in the variable domaine $\Omega_{t}=T_{t}(\Omega)$, where $\Omega_{t}=D \backslash \bar{\omega}_{t}$ and $\omega_{t}=T_{t}(\omega)$.

Taking the partial derivatives with respect to $t$ at $t=0$, and assuming that the shape derivatives of the test functions are null, we arrive at the identity

$$
\begin{gathered}
\int_{\partial \omega} \nabla u \cdot \nabla \varphi\langle V, n\rangle+\int_{\Omega} \nabla u^{\prime} \nabla \varphi+\int_{\partial \omega} u^{3} \varphi\langle V, n\rangle \\
+\int_{\Omega} 3 u^{2} u^{\prime} \varphi=\int_{\partial \omega} f \varphi\langle V, n\rangle,
\end{gathered}
$$

which can be written as follows

$$
\begin{gathered}
\int_{\Omega} \nabla u^{\prime} \nabla \varphi+\int_{\Omega} 3 u^{2} u^{\prime} \varphi \\
=-\int_{\partial \omega}\left[\nabla_{\Gamma} u \nabla_{\Gamma} \varphi+u^{3} \varphi-f \varphi\right]\langle V, n\rangle d \Gamma(x) .
\end{gathered}
$$

Now, we integrate by parts on the left hand side

$$
\int_{\Omega} \nabla u^{\prime} \nabla \varphi+\int_{\Omega} 3 u^{2} u^{\prime} \varphi=\int_{\Omega}\left(-\Delta u^{\prime}+3 u^{2} u^{\prime}\right) \varphi+\int_{\Gamma} \frac{\partial u^{\prime}}{\partial n} \varphi,
$$

which leads to the equation for $u^{\prime}$ in $\Omega$ provided that the test function $\varphi$ is compactly supported. In order to identify the Neumann boundary condition for $u^{\prime}$ on $\partial \omega$ we rewrite the boundary integrals

$$
\begin{aligned}
& \int_{\partial \omega}\left[-\nabla_{\Gamma} u \nabla_{\Gamma} \varphi+u^{3} \varphi-f \varphi\right]\langle V, n\rangle \\
= & \int_{\partial \omega}\left\{\operatorname{div}_{\Gamma}\left(\langle V, n\rangle \nabla_{\Gamma} u\right)+\left[u^{3}-f\right]\langle V, n\rangle\right\} \varphi,
\end{aligned}
$$

since the Green formula on the boundary takes the following form

$$
\int_{\partial \omega} a \nabla_{\Gamma} u \nabla_{\Gamma} \varphi d \Gamma=-\int_{\partial \omega} \varphi \operatorname{div}_{\Gamma}\left(a \nabla_{\Gamma} u\right),
$$

with $a=-V \cdot n$, we refer the reader to [32] for the details.

So we get

$$
\frac{\partial u^{\prime}}{\partial n}=\operatorname{div}_{\Gamma}\left(\langle V, n\rangle \nabla_{\Gamma} u\right)+\left[u^{3}-f\right]\langle V, n\rangle \quad \text { on } \quad \partial \omega .
$$

\section{B. Adjoint state}

The adjoint state is introduced in order to simplify the form of the shape gradient. Actually, the adjoint state equation is defined as follows

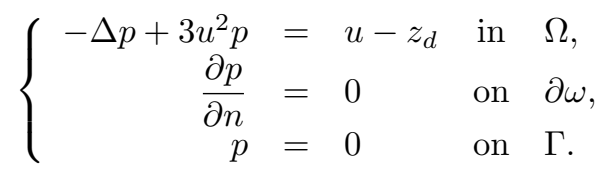

Variational formulation for the adjoint problem, taking into account the Green formula

$$
-\int_{\Omega} \Delta p u^{\prime}=\int_{\Omega} \nabla p \cdot \nabla u^{\prime}-\int_{\partial \Omega} \frac{\partial p}{\partial n} u,
$$

is as follows

$$
\int_{\Omega} \nabla p \cdot \nabla u^{\prime}+3 u^{2} p u^{\prime}=\int_{\Omega}\left(u-z_{d}\right) u^{\prime} .
$$

Now, we simplify the expression for the shape gradient, roughly speaking, the shape derivative is replaced by the 
adjoint state

$$
\begin{aligned}
\int_{\Omega}\left(u-z_{d}\right) u^{\prime} & =\int_{\Omega} \nabla p \cdot \nabla u^{\prime}+3 u^{2} p u^{\prime} \\
& =\int_{\Omega}\left[-\Delta u^{\prime}+3 u^{2} u^{\prime}\right] p+\int_{\partial \Omega} \frac{\partial u^{\prime}}{\partial n} p \\
& =\int_{\partial \omega}\left\{\operatorname{div}_{\Gamma}\left(\langle V, n\rangle \nabla_{\Gamma} u\right)+\left[u^{3}-f\right]\langle V, n\rangle\right\} p .
\end{aligned}
$$

As a result, the Eulerian semiderivative of the shape functional takes the form

$$
\begin{aligned}
d J(\Omega ; V)= & \int_{\partial \omega}\left(u-z_{d}\right)^{2}\langle V, n\rangle+\int_{\partial \omega} \operatorname{div}_{\Gamma}\left(\langle V, n\rangle \nabla_{\Gamma} u\right) p \\
& +\int_{\partial \omega}\left(u^{3}-f\right) p\langle V, n\rangle \\
= & \int_{\partial \omega}\left[\left(u-z_{d}\right)^{2}+\left(u^{3}-f\right) p\right]\langle V, n\rangle \\
& -\int_{\partial \omega}\langle V, n\rangle \nabla_{\Gamma} u \nabla_{\Gamma} p
\end{aligned}
$$

which leads to the following form of the shape gradient

$$
g_{\Gamma}=-\nabla_{\Gamma} u \cdot \nabla_{\Gamma} p+\left(u-z_{d}\right)^{2}+\left(u^{3}-f\right) p .
$$

\section{Topological derivative}

Topological derivative was developed in [8]. In this problem, the forme of topological derivative is obtained as follows:

$$
\begin{gathered}
\mathcal{T}(\mathcal{O})=-\pi\left[\left(u(\mathcal{O})-z_{d}(\mathcal{O})\right)^{2}+\nabla_{x} p(\mathcal{O})^{\top} \nabla_{n} u(\mathcal{O})\right. \\
\left.+f(\mathcal{O})-u(\mathcal{O})^{3}\right] .
\end{gathered}
$$

IV. NUMERICAL RESULTS FOR SHAPE AND TOPOLOGY OPTIMIZATION

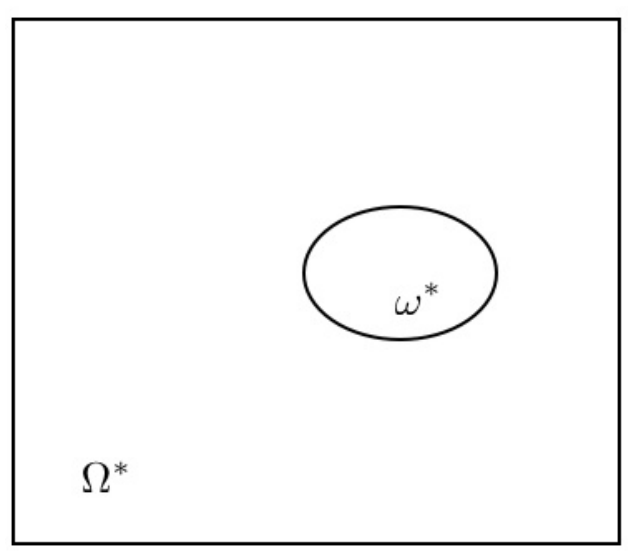

Fig. 2. Optimal domain

For the numerical results we use the tracking type shape functional (3) with a known element $z_{d}$ and a given optimal value. To this end an optimal hole $\omega^{*}$ is fixed and the following boundary value problem is considered for an auxiliary function $z$,

$$
\left\{\begin{array}{rlll}
-\Delta z+z^{3}=f & \text { in } & \Omega^{*} \\
z=0 & \text { on } & \Gamma \\
\frac{\partial z}{\partial n}=0 & \text { on } & & \partial \omega^{*}
\end{array} .\right.
$$

For the element

$$
z_{d}=\left\{\begin{array}{lll}
z & \text { in } & \Omega^{*} \\
0 & \text { in } & D \backslash \Omega^{*}
\end{array}\right.
$$

the optimal domain is $\Omega^{*}$ and the optimal value of the shape function is given by

$$
J\left(\Omega^{*}\right)=0
$$

\section{A. Numerical results}

For numerical calculations we consider the nonlinear problem (2) with the right hand side of the equation defined as

$$
\begin{aligned}
f\left(x_{1}, x_{2}\right)= & \left(\left(x_{1}^{2}+x_{2}^{2}\right) \sin \pi x_{1} \sin \pi x_{2}\right)^{3} \\
& +2\left(\pi^{2}\left(x_{1}^{2}+x_{2}^{2}\right)-2\right) \sin \pi x_{1} \sin \pi x_{2} \\
& -4 \pi\left(x_{1} \cos \pi x_{1} \sin \pi x_{2}+\sin \pi x_{1} \cos \pi x_{2}\right) .
\end{aligned}
$$

In the first example (Fig. 4) we consider the function $z_{d}$ which is defined in the domain $\Omega$ and is equal to zero in a circle of the center at $(0.7,0.7)$ and of the radius 0.5 .

As an initial domain we take a square without any hole. The evaluation of the topological derivative makes it possible to find the point where a small hole is placed. Next, the resulting domain is modify using the shape gradient. We get numerically the minimum of shape functional

$$
J\left(\Omega^{*}\right) \cong 0,000146 .
$$

In the second example (Fig. 6) we use the same functions $f$ and $z_{d}$ but we replace our initial domain by a square with a noncircular hole. In this case we get the minimal value of the objective function equal to:

$$
J\left(\Omega^{*}\right) \cong 0,000194
$$

\section{CONCLUSION}

Numerical method for the shape optimization of semilinear elliptic equations is proposed in the paper. The method is of the levelset type. The shape gradients of a tracking type functional are employed in the method as the coefficients of the Hamilton-Jacoby equations which governs the shape evolution. The topological derivatives are evaluated in order to change the topology of geometrical domain during the process of computations. The numerical results are presented and confirm that the method can be applied for numerical solution of the shape and topology optimization problem. 

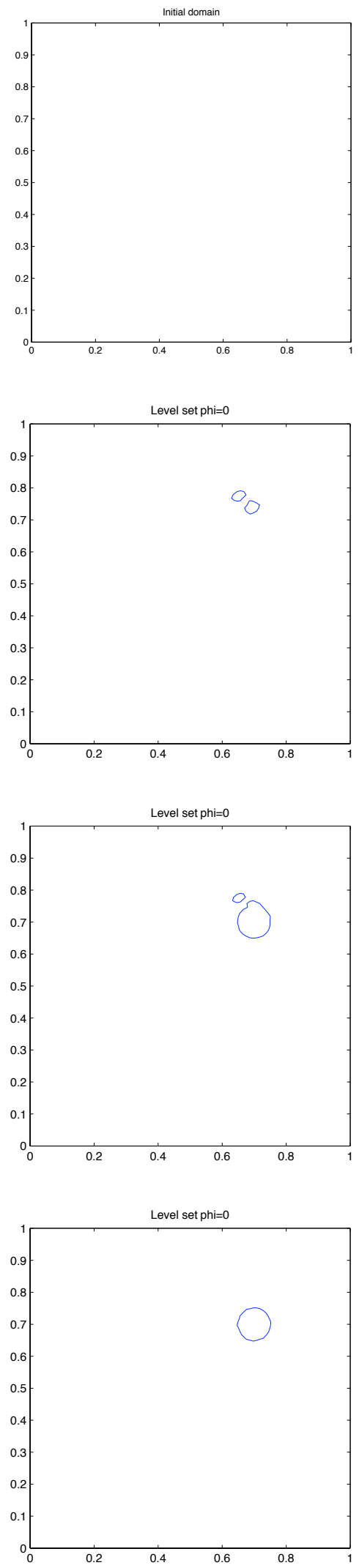

Fig. 3. Initial domain without hole. Evolution of the domain using topological derivative and shape gradient.

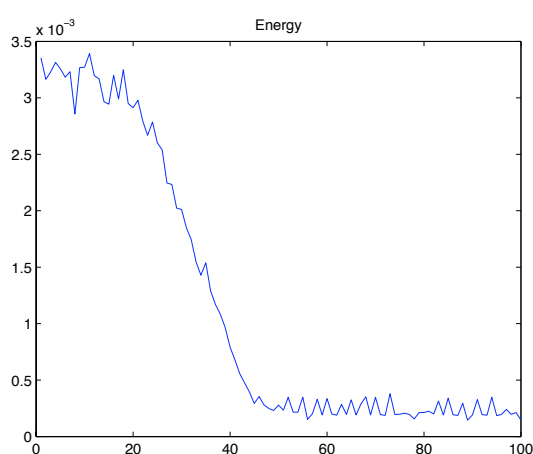

Fig. 4. Values of the objective function.

\section{REFERENCES}

[1] S. Amstutz, Topological sensitivity analysis for some nonlinear PDE system, J. Math. Pures Appl., 85, 2006, pp. 540-557.

[2] D. Bucur and G. Buttazzo, Variational methods in shape optimization problems, Progress in Nonlinear Differential Equations and their Applications, 65. Birkhauser Boston, Inc., Boston, MA, 2005.

[3] D. Bucur and N. Varchon, Boundary variation for a Neumann problem, Ann Scuola Norm.Sup.Pisa Cl.Sci., Vol. 4, 29, 2000, pp. 807-821.

[4] A. Demlow, Sharply localized pointwise and $W_{\infty}^{-1}$ estimates for finite element methods for quasilinear problems, Math. Comp. 76, 2007, pp. 1725-1741.

[5] J. Frehse, R. Rannacher, Asymptotic $L^{\infty}$-error estimates for linear finite element approximations of quasilinear boundary value problems, SIAM J. Numer. Anal., 15, 1978, pp. 418-431.

[6] P. Fulmanski, A. Lauraine, J.-F. Scheid, J. Sokołowski, A level set method in shape and topology optimization for variational inequalities, Int. J. Appl. Math. Comput. Sci., 2007, vol. 17, No. 3, pp. 413-430.

[7] E. J. Haug and J. Céa, Optimization of distributed parameter structures, Vol. I, II. Proceedings of the NATO Advanced Study Institute on Optimization of Distributed Parameter Structural Systems held in Iowa City, Iowa, May 20 - June 4, 1980. Edited by Edward J. Haug and Jean Céa. NATO Advanced Study Institute Series E: Applied Sciences, 49. Martinus Nijhoff Publishers, The Hague, 1981.

[8] M. Iguernane, S.A. Nazarov, J.-R. Roche, J. Sokolowski, K. Szulc, Topological derivatives for semilinear elliptic equations, Appl. Math. Comput. Sci., 2009, Vol. 19, No. 2, pp. 191-205.

[9] A. M. Il'in, Matching of asymptotic expansions of solutions of boundary value problems, Moscow: Nauka, [in Russian], 1989. [English translation: Matching of asymptotic expansions of solutions of boundary value problems. Translations of Mathematical Monographs, 102. American Mathematical Society, Providence, RI, 1992].

[10] L. Jackowska-Strumillo, J. Sokolowski, A. Zochowski and A. Henrot. Daly, On numerical solution of shape inverse problems, Comput. Optim. Appl. 23 (2002), pp. 231-255.

[11] V. A. Kondratiev, Boundary problems for elliptic equations in domains with conical or angular points, Trudy Moskov. Mat.Obshch. [in Russian], 16 (1967), pp. 2009-292. [English translasion: in Trans. Moscow Math. Soc. 16 (1967), pp. 227-313].

[12] O. A. Ladyzhenskaya, N. N. Ural'tseva, Linear and quasilinear elliptic equations, Academic Press, New York-London 1968 xviii+495 pp.

[13] N. S. Landkof, Fundamentals of modern potential theory, Izdat. "Nauka", Moscow [in Russian], 1966.

[14] A. Laurain, Domaines singulierements perturbes en optimisation de formes, Thèse de doctorat, http://tel.archivesouvertes.fr/docs/00/13/95/95/PDF/these5.pdf

[15] V. G. Mazja, S. A. Nazarov and B. A. Plamenevskii, On the asymptotic behavior of solutions of elliptic boundary value problems with irregular perturbations of the domain, Probl. Mat. Anal. Leningrad: Leningrad Univ. [in Russian], 8, 1981, pp. 72-153.

[16] V. G. Mazja, S. A. Nazarov and B. A. Plamenevskii, Asymptotische Theorie elliptischer Randwertaufgaben in singulär gestörten, Gebieten. 1. Berlin: Akademie-Verlag, [in Russian], 1991. [English translasion: Maz'ya V., Nazarov S., Plamenevskij B. Asymptotic theory of elliptic 

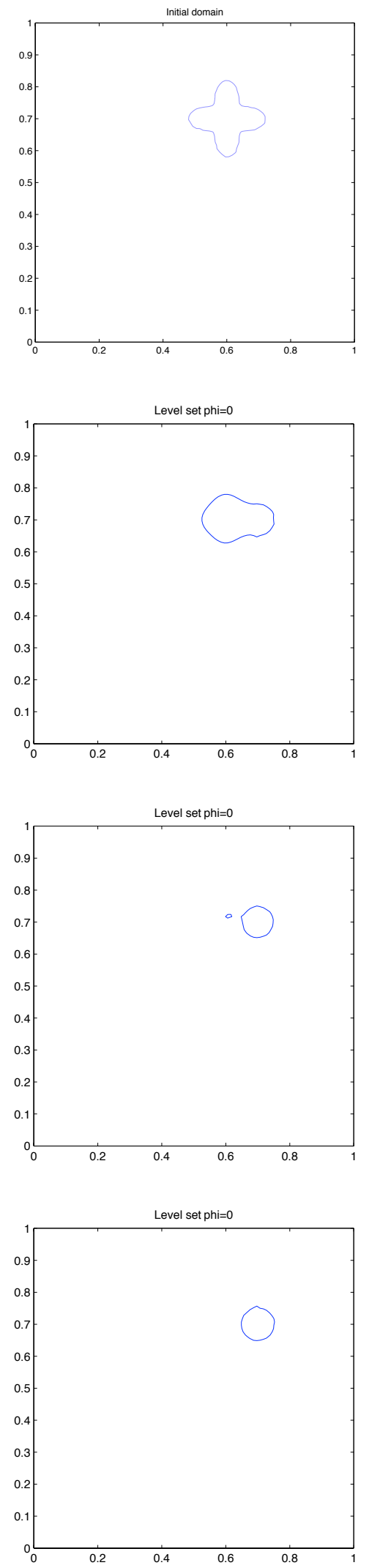

Fig. 5. Initial domain with a noncircular hole. Evolution of the domain using topological derivative and shape gradient.

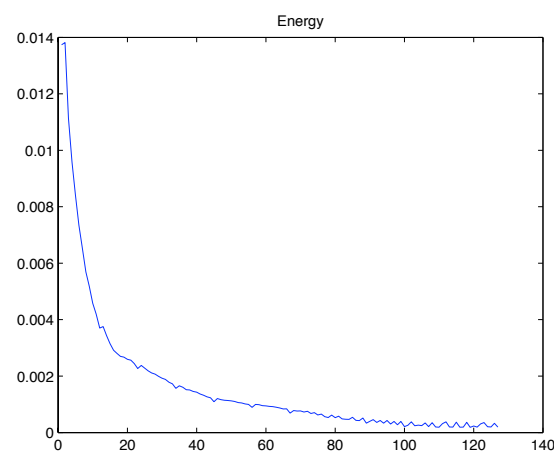

Fig. 6. Values of the objective function.

boundary value problems in singularly perturbed domains. Vol. 1, Basel: Birkhäuser Verlag, 2000.

[17] V. G. Mazja and B. A. Plamenevskii, Estimates in $L_{p}$ and Hölder classes and the Miranda-Agmon maximum principle for solutions of elliptic boundary value problems in domains with singular points on the boundary, Math. Nachr. [in Russian], 81 (1978), pp. 25-82. [English translasion in: Amer. Math. Soc. Transl. (Ser. 2) 123 (1984), pp. 1-56]

[18] V. G. Mazja and B. A. Plamenevskii, On the behavior of solutions to quasilinear elliptic boundary-value problems in a neighborhood of a conical point. Zap. Nauchn. Sem. LOMI, 38 (1973), pp. 91-97.

[19] S. A. Nazarov and B. A. Plamenevsky, Elliptic Problems in Domains with Piecewise Smooth Boundaries. De Gruyter Exposition in Mathematics 13, Walter de Gruyter, 1994.

[20] S. A. Nazarov and J. Sokolowski, Self-adjoint extensions for the Neumann Laplace and applications. Acta Math. Sin. 22 (2006), pp. 879906.

[21] S. A. Nazarov and J. Sokolowski, Asymptotic analysis of shape functional. J. Math. Pures Appl. 82 (2003), pp. 125-196.

[22] S. Osher, R. Fedkiw, Level set methods and dynamic implicit surfaces, Springer, 2004.

[23] D. Peng, B. Merriman, S. Osher, H. Zhao, M. Kang, A PDE-based fast local level set method, J. Comp. Phys., 1999, Vol.155, p.410-438.

[24] G. Pólya and G. Szegö, Isoperimetric inequalities in mathematical phisics. Princeton University Press, Princeton, NJ, 1951.

[25] P. A. Raviart and J. M. Thomas, Introduction à l'analyse numérique des équations aux dérives partielles. Masson, Paris, 1983.

[26] J. Sokolowski and A. Zochowski, Modelling of topological derivatives for contact problems. Numer. Math. 102 (2005), pp. 145-179.

[27] J. Sokolowski and A. Zochowski, Introduction to shape optimization. Shape sensitivity analysis. Springer Series in Computational Mathematics, Springer-Verlag, Berlin, 16 (1992).

[28] J. Sokolowski and A. Zochowski, On topological derivative in shape optimization. SIAM Journal on Control and Optimization. 37 (1999), pp. 1251-1272.

[29] J. Sokolowski and A. Zochowski, Optimality conditions for simultaneous topology and shape optimization. SIAM Journal on Control and Optimization, 42 (2003), pp. 1198-1221.

[30] J. Sokolowski and A. Zochowski, Topological derivatives of shape functional for elasticity systems. Mech. Structures Mach. 29 (2001), pp. 331-349.

[31] J. Sokolowski and A. Zochowski, On the topological derivative in shape optimization. SIAM J. Control Optim. 37 (1999), pp. 1251-1272.

[32] J. Sokołowski, J.-P.Zolésio, Introduction to shape optimization. Shape sensitivity analysis. Springer-Verlag, 1992, New York.

[33] G. Stampacchia, Le problème de Dirichlet pour les équations elliptiques du second ordre à coefficients discontinus. Ann. Inst. Fourier (Grenoble), 15 (1965), pp. 189-258.

[34] K. Szulc, Quelques méthodes numeriques en opitmisation de formes, Thèse de doctorat.

[35] H.K. Zhao, T. Chan, B. Merriman, S. Osher A variational level set approach to multi-phase motion. J. Comp. Phys., 1996, Vol.122, p.179195. 